\title{
Synthesis, Characterization and Photocrosslinkable Studies of Certain Thermotropic Liquid Crystalline Poly(ester-amides)
}

\author{
Kavitha Erra Kalappa, S. Arul Antony, N. Ramalakshmi* \\ Post-Graduate and Research Department of Chemistry, Presidency College (Autonomous), Chennai - 600 005, Tamil Nadu, India.
}

\section{ART ICLE DETAILS}

\section{Article history:}

Received 15 June 2018

Accepted 27 June 2018

Available online 22 July 2018

\section{Keywords:}

Bisbenzylidenecyclohexanone

Poly(ester-amides)

Polycondensation

Photocrosslinkability

\begin{abstract}
A B S T R A C T
Five thermotropic liquid crystalline poly(ester-amides) were synthesized by polycondensation method using varying dicarboxylic acids with a common diamine namely 4,4'-diaminodiphenyl and a common diol namely 2,6-bis(4-hydroxy-3-methoxybenzylidene)cyclohexanone (CHBV). The solubility data and viscosity measurements of these synthesized poly(ester-amides) were used for qualitative characterization. The spectroscopic techniques such as FT-IR, ${ }^{1} \mathrm{H}$ NMR, ${ }^{13} \mathrm{C}$ NMR were performed for investigating micro structural features. By differential scanning calorimetry (DSC), the thermal phase transition behavior of these poly(ester-amides) was studied. Scanning electron microscopy (SEM) technique was used to investigate the morphology of these poly(ester-amides). By using UV-visible spectroscopic technique, the photocrosslinkability of these poly(ester-amides) were studied by irradiating these poly(ester-amides) under a $160 \mathrm{~W}$ medium pressure mercury lamp. SEM analysis of these UV irradiated poly(ester-amides) showed the changes in morphology. Thus, the poly(esteramides) containing arylidene keto moiety in the main chain exhibited nematic mesophase and were photocrosslinked upon UV irradiation. X-ray diffraction (XRD) patterns of these poly(ester-amides) were taken to assess the degree of crystallinity.
\end{abstract}

\section{Introduction}

The bisbenzylidene cyclohexanone has both mesogenic and photoactive properties. This can exist in three isomeric structures $E E, E Z$ and $Z Z$. Among these three isomers, $E E$ has a linear rod shaped structure. Synthesis of bisbenzylidene cycloalkanone molecule was first reported by Samdahl $[1,2]$. This photo-active molecule has attracted the attention of macromolecular chemists [3-7]. They were found to be a potential mesogen [8-10] and their incorporation in the polymeric backbone has imparted thermotrophic liquid crystalline property to the polymeric materials. Thermotropic liquid crystalline polymers have wide range of applications from ultrahigh strength fibres to nonlinear optical devices [11-13]. Polyesters synthesized have been reported to possess intriguing anisotropic, mechanical, electrical and optical properties suitable for technological applications [14]. Photocrosslinkable polymers have found applications in microlithography including printing technology, integrated circuit technology, photoconductors, photofabrication, photocrosslinked hydrogels etc [15-17]. Kannappan et al [18] were investigated the rate of photocrosslinking behavior of random copolyesters with arylidene-keto moiety by UV spectral studies. Even the poly(ester-amides) was synthesized by making use of arylidene-keto moieties $[19,20]$.

However, there is no literature information found on the synthesis and photocrosslinkability of poly(ester-amides) containing bis(arylidene) cyclohexanone moiety in the polymer backbone. The purpose of the current work is to synthesize five poly(ester-amides) containing bis(arylidene)cyclohexanone moiety in the polymer backbone and to evaluate its photocrosslinkability. Poly(ester-amides) are a category of polymeric materials which contains both ester and amide linkages [21,22] and are synthesized by the copolymerization of a diacid with that of a diamine and a diol in the mole ratio of 2:1:1.

\section{Experimental Methods}

\subsection{Chemicals}

Aldrich samples of terephthalic acid, isophthalic acid, phthalic acid, adipic acid, azelaic acid, diphenyl chlorophosphate, and benzidine were used as received. Lithium chloride (SD Fine), vanillin (CDH), and cyclohexanone (Fluka) were used as received. Pyridine (Merck) was used as polymerisation medium was refluxed over potassium hydroxide pellets, distilled (b.p. $115^{\circ} \mathrm{C}$ ) and stored over potassium hydroxide pellets. Methanol (Merck, LR) was used as solvent. N,N-dimethylacetamide (DMAc) (SD-Fine, AR) was used as solvent for finding out the inherent viscosity and UV-visible spectra of the poly(ester-amide). DMSO-d6 (Aldrich spectral grade) was used for recording NMR spectra.

\subsection{Preparation of Monomer \\ 2.2.1 Synthesis of Diol}

The arylidene-keto diol, 2,6-bis(4-hydroxy-3-methoxybenzylidene) cyclohexanone (CHBV) were synthesized by the method reported in literature $[23,24]$.

Preparation of 2,6-bis(4-hydroxy-3-methoxybenzylidene)cyclohexanone (CHBV)

Vanillin(4-hydroxy-3-methoxybenzaldehyde) (0.2 mol) in $100 \mathrm{~mL}$ of dry methanol was taken in a $250 \mathrm{~mL}$ conical flask. Drop wise cyclohexanone $(0.1 \mathrm{~mol})$ was added and shaken well. Fuming sulphuric acid $(5 \mathrm{~mL})$ with external cooling in an ice bath was added drop wise. The reaction mixture turned bright yellow, dark green and finally pink due to an exothermic reaction. The precipitated diol was filtered and washed with aqueous methanol. It was filtered and recrystallised from aqueous methanol. Yield: 95\% (m.p.: $180^{\circ} \mathrm{C}$ ).
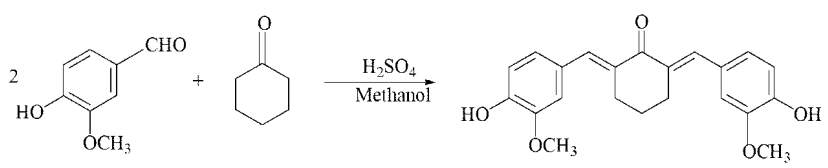

Vanillin 


\subsubsection{Synthesis of Poly(ester-amides)}

Two methods were employed to synthesize the poly(ester-amides) depending upon the type of dicarboxylic acid monomer (aromatic or aliphatic).

Method 1: Synthesis of Poly(ester-amides) Derived from Aromatic Dicarboxylic Acid Monomer

Arul Moli and coworkers [25] were reported the procedure for the synthesis of a typical poly(ester amide) and are represented here.

By using diphenyl chlorophosphate (DPCP) as condensation agent, five poly(ester-amides) were prepared by direct polycondensation of a diamine, a diol with one diacid in the respective mole ratio 1:1:2 in pyridine solution. This method avoids the tedious preparation of acid chloride and the yield of the polymer is also high.

In a $100 \mathrm{~mL}$ round-bottomed flask, $\mathrm{LiCl}(10 \mathrm{mmol})$ and aromatic dicarboxylic acid $(5 \mathrm{mmol})$ in pyridine $(10 \mathrm{~mL})$ were taken and diphenyl chlorophosphate $(12 \mathrm{mmol})$ in pyridine $(10 \mathrm{~mL})$ was added to it. At room temperature, the reaction mixture was continuously stirred for a span of 30 minutes. Then the temperature was raised to $115^{\circ} \mathrm{C}$ and stirring was carried out at this temperature for about 10 minutes. The solution containing the diamine diphenyl diamine $(2.5 \mathrm{mmol})$ and the diol $(2.5$ $\mathrm{mmol}$ ) was added over a period of 10 minutes with constant stirring. The reaction mixture was maintained at this temperature for a time frame of 3 hours. Then reaction mixture was cooled to room temperature and poured into $300 \mathrm{~mL}$ of methanol when the poly(ester-amide) was precipitated. It was filtered, washed with methanol and dried in vacuum.

Method 2: Synthesis of Poly(ester-amides) Derived from Aliphatic Diacid Chloride Monomer

The procedure for the synthesis of a typical aliphatic diacid-based polymer is given here. Four aliphatic diacid-based poly(ester-amides) were prepared by the polycondensation of a diamine, a diol with one diacid in the respective mole ratio of 1:1:2 in DMF [26].

The common diamine diphenyldiamine $(2.5 \mathrm{mmol})$ and the diol $(2.5$ mmol) were dissolved in DMF $(30 \mathrm{~mL})$ were taken in a $100 \mathrm{~mL}$ roundbottomed flask. Diacid chloride (5 mmol) was added drop wise with stirring to the solution at $100{ }^{\circ} \mathrm{C}$. The reaction mixture was heated at 115 ${ }^{\circ} \mathrm{C}$ for 8 hours with stirring at nitrogen atmosphere. The contents were cooled and poured into methanol. The mixture was kept in a refrigerator overnight and then filtered. The poly(ester-amide) was dissolved in minimum amount of acetone, filtered and the clear solution was poured into water. The precipitated poly(ester-amide) was dried in vacuum over phosphorus pentoxide.

The various poly(ester-amides) prepared by these methods and the monomers are given in Table 1 along with the polymer code, percentage of yield and inherent viscosities.

Table 1 Monomers used and the polymer code of poly(ester-amides) with percentage of yield and inherent viscosities $\left(\eta_{\text {inh }}\right)$

\begin{tabular}{|c|c|c|c|c|c|}
\hline \multirow{2}{*}{ S. No. } & \multicolumn{2}{|c|}{ Common diamine: diphenyl diamine } & \multirow{2}{*}{$\begin{array}{l}\text { Polymer } \\
\text { Code }\end{array}$} & \multirow{2}{*}{$\begin{array}{l}\text { Yield } \\
(\%)\end{array}$} & \multirow{2}{*}{$\begin{array}{l}\eta_{\text {inh }} \\
(\mathrm{dL} / \mathrm{g})\end{array}$} \\
\hline & Diol & Diacid / Diacid chloride & & & \\
\hline 1. & CHBV & Terephthalic acid & PVDT & 78 & 1.23 \\
\hline 2. & CHBV & Isophthalic acid & PVDI & 76 & 1.19 \\
\hline 3. & $\mathrm{CHBV}$ & Phthalic acid & PVDP & 75 & 1.18 \\
\hline 4. & CHBV & Adipoyl dichloride & PVDA & 60 & 0.59 \\
\hline 5. & $\mathrm{CHBV}$ & Azelaoyl dichloride & PVDAz & 62 & 0.62 \\
\hline
\end{tabular}

CHBV: 2,6-bis(4-hydroxy-3-methoxybenzylidene)cyclohexanone

\subsection{Characterization Methods}

All the poly(ester-amides) was characterized by solubility studies, viscosity measurements, and spectral data. Thermal investigations were made on these poly(ester-amides) by differential scanning calorimetry. X-ray diffraction (XRD) patterns were taken to assess the degree of crystallinity in these poly(ester-amides). The morphology of these poly(ester-amides) was established with SEM analysis. The photocrosslinkable behavior of these poly(ester-amides) was studied by UV-visible spectra.

\subsubsection{Solubility}

The solubility of these poly(ester-amides) was tested in various solvents qualitatively. About $50 \mathrm{mg}$ of the polymer was taken in a small stoppered test tube containing $1 \mathrm{~mL}$ of the solvent. The mixture was kept for 24 hours with occasional shaking.

\subsubsection{Viscosity}

The inherent viscosity $\left(\eta_{\text {inh }}\right)$ of the five poly(ester-amides) was determined in $\mathrm{N}, \mathrm{N}$-dimethylacetamide (DMAc) solution at $30{ }^{\circ} \mathrm{C}$ using Ubbelohde viscometer in which the pure solvent had a flow rate of 470 seconds. In each case, $25 \mathrm{mg}$ of dry poly(ester-amides) sample was dissolved in $25 \mathrm{~mL}$ of DMAc, kept aside for 12 hours with occasional shaking. The $\eta_{\text {inh }}$ was calculated from the flow time measurement at $30^{\circ} \mathrm{C}$.

\subsubsection{Fourier Transform Infrared (FT-IR) Spectroscopy}

The FT-IR spectra were recorded using Shimadzu FT-IR instrument in the form of potassium bromide pellet method.

\subsection{4 ${ }^{1} \mathrm{H}$ and ${ }^{13} \mathrm{C}$ NMR Spectra}

The ${ }^{1} \mathrm{H}$ and ${ }^{13} \mathrm{C}$ NMR spectra were recorded with Bruker AVANCE III 500 MHz instrument in DMSO-d6 solvent with TMS as internal reference.

\subsubsection{Differential Scanning Calorimetry (DSC)}

In thermal analysis of these poly(ester-amides), DSC thermograms were recorded in NETZSCH DSC 200F3 differential scanning calorimeter using $5 \mathrm{mg}$ samples under nitrogen atmosphere at a heating rate of 10 ${ }^{\circ} \mathrm{C} / \mathrm{min}$.

\subsubsection{X-Ray Diffraction (XRD)}

The X-ray diffraction measurements were taken to assess the degree of crystallinity of these powdered poly(ester-amides) using GE-Inspection Technology Diffractometer System XRD 3003 TT model made in Germany with a source of copper target operating voltage $40 \mathrm{Kv} 300 \mathrm{mÅ}$ current rate.

\subsubsection{Photocrosslinking Studies}

The UV-visible spectra were recorded using Shimadzu UV-160A instrument in DMAc solvent. The photocrosslinkable behavior of these poly(ester-amides) were recorded by irradiating these poly(ester-amides) under a $160 \mathrm{~W}$ medium pressure mercury lamp.

\subsubsection{Scanning Electron Microscopy (SEM)}

The morphology of these poly(ester-amides) was investigated before and after UV irradiation under a $160 \mathrm{~W}$ medium pressure mercury lamp using Hitachi S-3000 Hz scanning electron microscopy (SEM).

\section{Results and Discussion}

The poly(ester-amides) synthesized in the present work were characterized by solubility studies, viscosity measurements and spectral data. X-ray diffraction (XRD) patterns were taken to assess the degree of crystallinity in these poly(ester-amides). Thermal analysis was established to study the phase transition behavior. The nematic mesophase and photocrosslinkable behavior of these poly(ester-amides) were investigated before and after UV irradiation under a $160 \mathrm{~W}$ medium pressure mercury lamp using SEM and UV-visible analysis.

\subsection{Solubility}

The poly(ester-amides) reported here are found to be soluble in highly polar solvents such as dimethyl sulphoxide, DMAc and trifluoroaceticacid, partially soluble in moderately polar solvents and thoroughly insoluble in non-polar solvents such as benzene and hexane. This might be recognized due to the inter-molecular interactions of polar solvents with ether linkages of the polymer backbone. Similar explanation was offered by Sathish and coworkers [27] in a series of copolyesters. Also, the poly(ester-amides) with methoxy substituent in the phenyl ring of the arylidenecyclohexanone moiety was found to be highly soluble because of their capacity to disrupt the macromolecular polymer chain molecules. The results of the solubility of the poly(ester-amides) are represented in Table 2.

Table 2 Solubility of poly(ester-amides) in common organic solvents

\begin{tabular}{llllllllllllll}
\hline S.No. & Polymer Hexane & Benzene & $\mathrm{CHCl}_{3}$ & EtOAc & THF & \multicolumn{1}{ll}{ Acetone DMF } & $\mathrm{CH}_{3} \mathrm{CN}$ & $\mathrm{DMAc}$ & $\mathrm{TFA}$ & $\mathrm{DMSO}$ \\
\hline 1 & PVDT & -- & -- & -- & -- & +- & +- & ++ & ++ & ++ & ++ & ++ \\
2 & PVDI & -- & -- & -- & -- & +- & +- & ++ & ++ & ++ & ++ & ++ \\
3 & PVDP & -- & -- & -- & -- & +- & +- & ++ & ++ & ++ & ++ & ++ \\
4 & PVDA & -- & -- & +- & +- & ++ & ++ & ++ & ++ & ++ & ++ & ++ \\
5 & PVDAz & -- & -- & +- & +- & ++ & ++ & ++ & ++ & ++ & ++ & ++ \\
\hline
\end{tabular}

$++=$ Freely Soluble; -- = Insoluble; +- = Partially soluble 


\subsection{Viscosity}

The $\eta_{\text {inh }}$ values of all the five poly(ester-amides) were found to be in the range of $0.59-1.23 \mathrm{dL} / \mathrm{g}$ and are represented in Table 1 . It may be pointed out that the poly(ester-amides) synthesized from aromatic dicarboxylic acids have higher $\eta_{\text {inh }}$ values than those prepared from aliphatic diacid chloride monomers. The data shows higher viscosity values and hence they are reasonably of high molecular weight.

\subsection{Spectral Studies}

The ester and amide functional groups present in the poly(ester-amide) chain were identified by FT-IR spectra. The IR spectra of all the five poly(ester-amides) showed characteristic absorption at $\bar{v}=1630-1750$ $\mathrm{cm}^{-1}$ due to ester and amide $\mathrm{C}=0$ stretching frequency and an absorption at $\bar{v}=3280-3380 \mathrm{~cm}^{-1}$ due to the amide $\mathrm{N}-\mathrm{H}$ stretching frequency. Similar observations were made by Khairou et al [19] in a new series of poly(esteramides) containing diarylidenecyclohexanone in the main chain. They are represented in Figs. 1(a) and (b) for PVDT and PVDA respectively.

The structural units present in the poly(ester-amide) chain were identified by ${ }^{1} \mathrm{H}$ and ${ }^{13} \mathrm{C}$ NMR spectra. The secondary amide proton appeared as a singlet in the range of $9.5-10.15 \mathrm{ppm}$. Related observation was made by Kannapan and Reuben Jonathan [20]. The aromatic protons are observed in the range of 6.5-8.54 ppm. The methoxy protons in the vanillin moiety are indicated by a signal at $3.8 \mathrm{ppm}$. They are represented in Figs. 2(a) and (b) for PVDT and PVDA respectively.

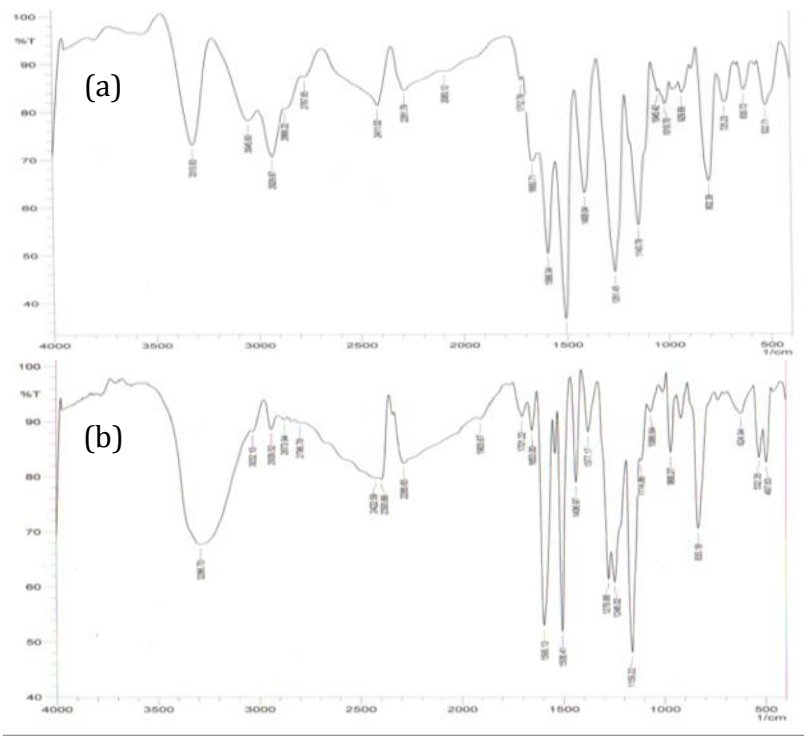

Fig. 1 FT-IR spectrum of random poly(ester-amides) (a) PVDT and (b) PVDA

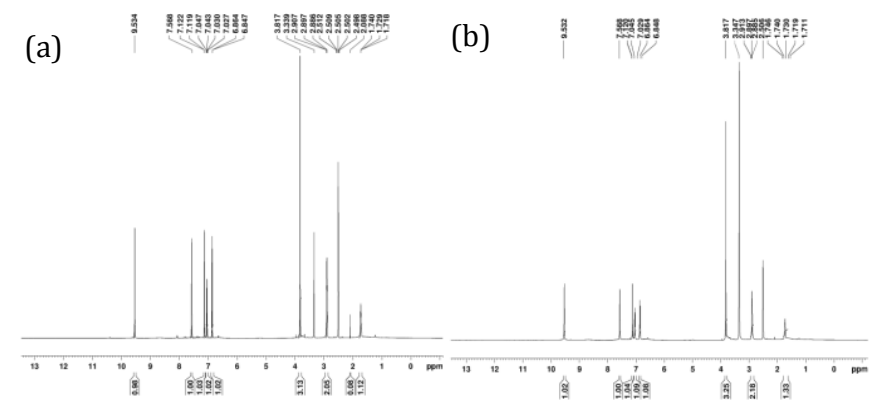

Fig. $2{ }^{1} \mathrm{H}$ NMR spectrum of random poly(ester-amides) (a) PVDT and (b) PVDA

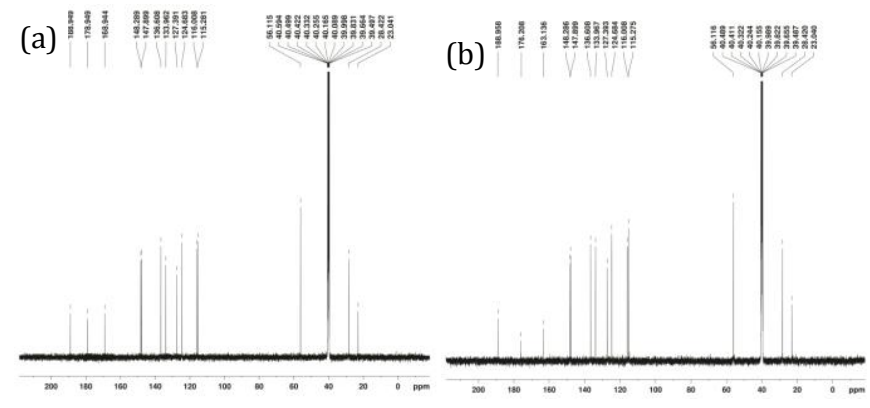

Fig. $3{ }^{1} \mathrm{C}$ NMR spectrum of random poly(ester-amides) (a) PVDT and (b) PVDA
The signal in the range of $\delta 188-200 \mathrm{ppm}$ in the ${ }^{13} \mathrm{C}$ NMR spectra of the poly(ester-amides) is due to the carbonyl carbon of the $\alpha, \beta$-unsaturated ketone. The signals in the range of $\delta 171-185 \mathrm{ppm}$ and $\delta 160-170 \mathrm{ppm}$ are due to the carbonyl carbon of the amide and ester groups [19] which indicates the formation of poly(ester-amide). They are represented in Figs. 3(a) and (b) for PVDT and PVDA respectively.

\subsection{Thermal Analysis and Phase Behavior}

The thermal and phase behaviors of the five poly(ester-amides) were investigated by differential scanning calorimetry (DSC). The glass transition temperature $\left(\mathrm{T}_{\mathrm{g}}\right)$, crystal to crystal transition $\left(\mathrm{T}_{\mathrm{K} \rightarrow \mathrm{K}}\right)$, melting temperature $\left(\mathrm{T}_{\mathrm{m}}\right)$, isotropization temperature $\left(\mathrm{T}_{\mathrm{i}}\right)$ and liquid crystalline range are tabulated in Table 3.

Table 3 Differential scanning calorimetry (DSC) data of poly(ester-amides)

\begin{tabular}{lllllll}
\hline \multirow{2}{*}{ S.No. } & \multirow{2}{*}{ Polymers } & \multicolumn{6}{l}{ Differential scanning calorimetry (DSC) } \\
\cline { 3 - 7 } & & $\mathrm{T}_{\mathrm{g}}\left({ }^{\circ} \mathrm{C}\right)$ & $\mathrm{T}_{\mathrm{K} \rightarrow \mathrm{K}}\left({ }^{\circ} \mathrm{C}\right)$ & $\mathrm{T}_{\mathrm{m}}\left({ }^{\circ} \mathrm{C}\right)$ & $\mathrm{T}_{\mathrm{i}}\left({ }^{\circ} \mathrm{C}\right)$ & $\Delta \mathrm{T}$ \\
\hline 1 & PVDT & 22.5 & 180.0 & 330.0 & 460.0 & 130.0 \\
2 & PVDI & 24.0 & 178.5 & 336.0 & 438.5 & 102.5 \\
3 & PVDP & 23.3 & 163.3 & 325.8 & 393.3 & 67.5 \\
4 & PVDA & 32.9 & 175.4 & 312.9 & 477.9 & 165.0 \\
5 & PVDAz & 25.4 & 180.4 & 300.4 & 390.4 & 90.0 \\
\hline
\end{tabular}

The DSC thermograms of all poly(ester-amides) are shown in Fig. 4. The analysis of DSC data shows that the poly(ester-amides) synthesized from aromatic dicarboxylic acids have lower glass transition temperature $\left(\mathrm{T}_{\mathrm{g}}\right)$ values than those prepared from aliphatic diacid chloride monomers. In these polymers, an exothermic peak appears before melting transition, which seems to be related to crystal to crystal transition resulted from different crystalline polymorphs [28]. The poly(ester-amide) derived from adipic acid monomer maintain broader liquid crystalline range $(\Delta \mathrm{T})$ than the poly(ester-amide) derived from azelaic acid monomer. This may be due to the coplanar geometry that would favor effective molecular packing. Also, while comparing the liquid crystalline range $(\Delta T)$ of PVDT, PVDI, and PVDP, the poly(ester-amide) derived from terephthalic acid monomer maintain broader liquid crystalline range $(\Delta T)$. This may be due to the rigidity of p-phenylene moiety when compared to m-phenylene or o-phenylene moiety in the polymer chain. It is observed that these poly(ester-amides) have higher $\mathrm{T}_{\mathrm{g}}$ values which could be due to interlocking effect of the methoxy substituent present in the arylidene keto moiety. There are reports on such interlocking effects on the thermal properties of polymers by Lenz and coworkers [11]. They suggested that the interlocking effect depends on the size of the substituent. This was further supported by reported work of Kannappan and coworkers [24] by ultrasonic method.

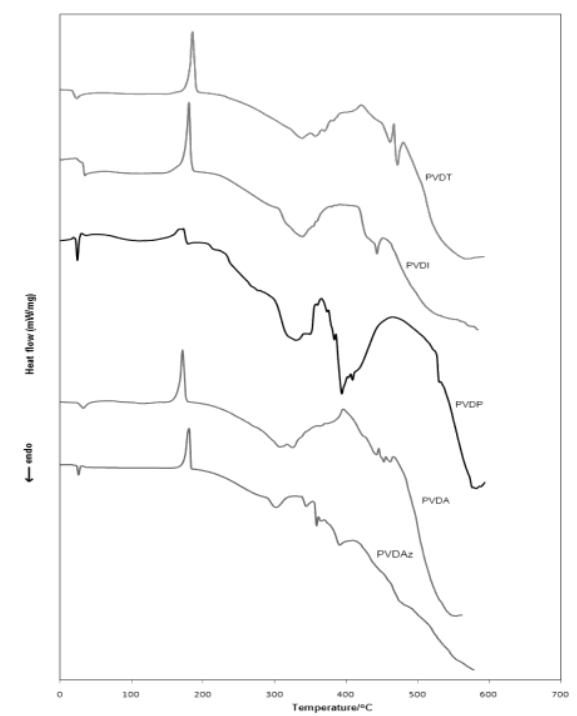

Fig. 4 DSC thermograms of random poly(ester-amides)

\subsection{X-Ray Diffraction Studies}

The X-Ray diffraction pattern of poly(ester-amides) PVDT and PVDA are shown in Figs. 5(a) and (b) respectively. It shows reflection peaks in the region $2 \theta=10-50^{\circ}[19]$ which indicates their semicrystallinity with an amorphous background. The presence of $\mathrm{C}=\mathrm{O}$ and $\mathrm{C}=\mathrm{C}$ groups induces some degree of crystallinity. But the presence of methoxy substituent in the benzylidene moiety caused some hindrance between repeating units leading to unsymmetrical orientation and reduced the crystallinity. 

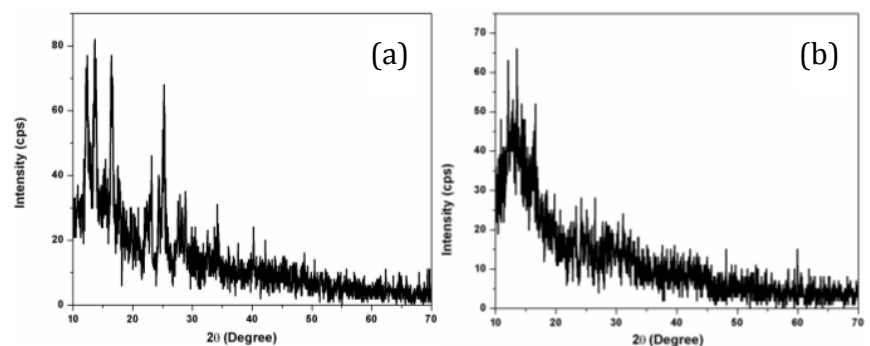

Fig. 5 X-ray diffraction pattern of random poly(ester-amides) (a) PVDT and (b)PVDA

\subsection{Photocrosslinking Studies}

The poly(ester-amides) containing the arylidene keto moiety shows the dual function of a mesogen and a photo-active chromophore and undergoes photocrosslinking on exposure to UV irradiation. The photocrosslinkability was established by recording UV-visible spectra for the poly(ester-amides) in the concentration range of $10-20 \mathrm{mg} / \mathrm{L}$ in DMAc solution before and after UV irradiation under a $160 \mathrm{~W}$ medium pressure mercury lamp for $300 \mathrm{~min}$. The $\pi \rightarrow \pi^{*}$ transition of the exocyclic double bond in bis(3-methoxybenzylidene)cyclohexanone moiety resulted in the absorption around 300-400 $\mathrm{nm}$. The decrease in absorbance (A) with time after UV irradiation, indicates that there is photocrosslinking behavior on irradiation. This is due to the $2 \pi+2 \pi$ cycloaddition reactions of $\mathrm{C}=\mathrm{C}$ bonds in the arylidene unit, leading to the formation of cyclobutane ring. The UVvisible spectra of the poly(ester-amides) PVDT and PVDA before and after UV irradiation are shown in Figs. 6(a) and (b) respectively.

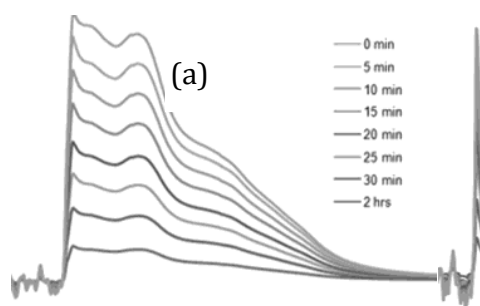

(b)

Fig. 6 UV-visible spectrum of random poly(ester-amides) (a) PVDT and (b) PVDA

To compare the relative reactivity of these polymers, a plot of $\left(A_{0}-A_{t} / A_{0}\right)$ against time was obtained, where $\mathrm{A}_{0}$ is the absorbance before irradiation and $A_{t}$ is the absorbance after irradiation for time $t$. They are shown in Figs. 7(a) and (b) for PVDT and PVDA respectively. Perundevi and coworkers [29] were reported on the steady rate of photocrosslinking behavior for certain photocrosslinkable random copolyesters.
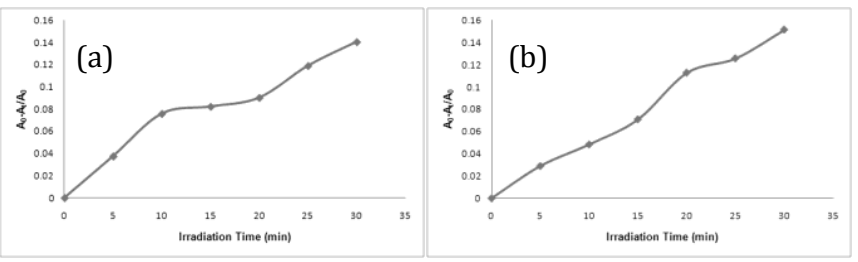

Fig. 7 Relative reactivity of random poly(ester-amides) (a) PVDT and (b) PVDA

The rate of disappearance of $>\mathrm{C}=\mathrm{C}<$ of the arylidene keto moiety of the five poly(ester-amides) was determined by measuring the UV absorption intensity of the solution after each exposure interval using the following expression:

$$
\text { Rate of conversion }(\%)=\frac{A_{0}-A_{t}}{A_{0}} \times 100
$$

It is shown in Fig. 8. Suresh and coworkers [30] were reported on the rate of photocrosslinking behavior for certain polymers.

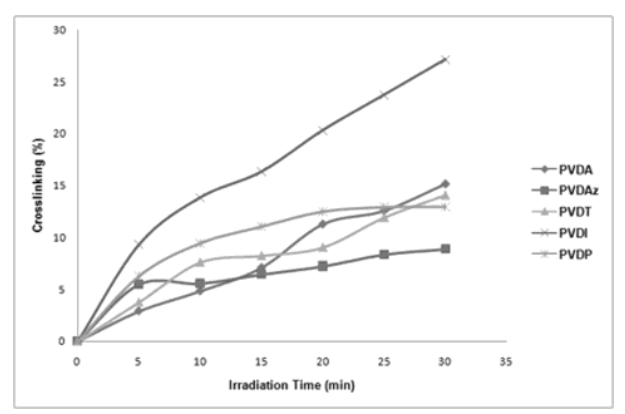

Fig. 8 Crosslinking percentage of random poly(ester-amides)

\subsection{Morphological Study by SEM}

The SEM images of the poly(ester-amides), before and after irradiation to UV light illustrates the changes in the morphology due to photocrosslinking. They are represented in Figs. 9 and 10 for PVDT and PVDA respectively.
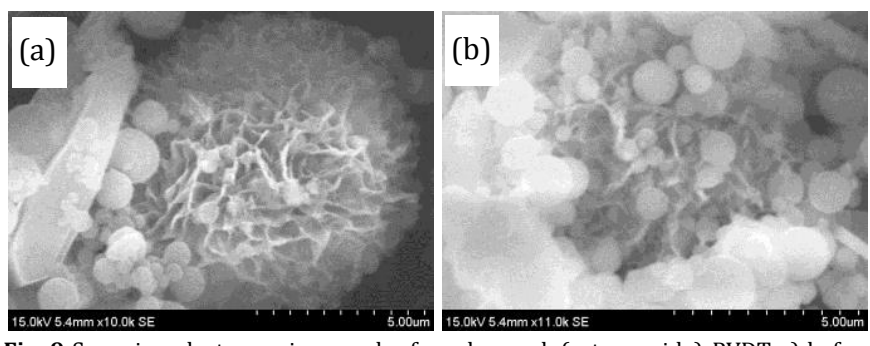

Fig. 9 Scanning electron micrograph of random poly(ester-amide) PVDT a) before and b) after irradiation to UV light

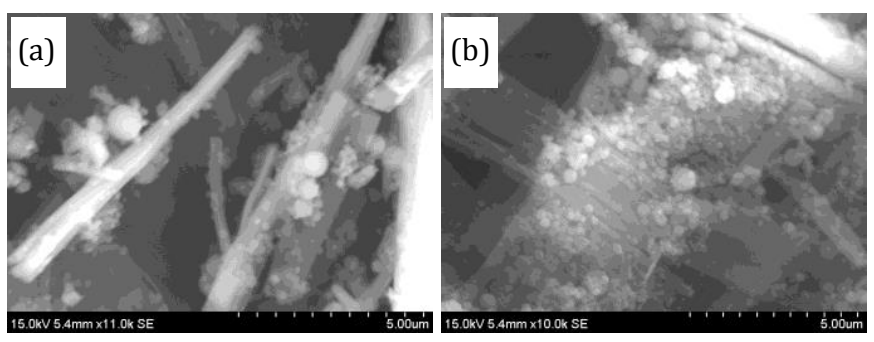

Fig. 10 Scanning electron micrograph of random poly(ester-amide) PVDA a) before and b) after irradiation to UV light

\section{Conclusion}

The five poly(ester-amides) have been synthesized successfully by direct polycondensation method. These poly(ester-amides) are highly soluble in polar organic solvents. The viscosities measurements reveal that the poly(ester-amides) synthesized are of high molecular weight. The synthesized poly(ester-amides) has been characterized by FT-IR, ${ }^{1} \mathrm{H}-\mathrm{NMR}$, and ${ }^{13} \mathrm{C}-\mathrm{NMR}$ spectral studies. The structural assignment of these poly(ester-amides) is supported by these spectral data. The liquid crystalline property of these poly(ester-amides) was recognized from DSC. The study of photocrosslinking behavior of these poly(ester-amides) reveals their requisite importance in photo-resist applications. The poly(ester-amides) synthesized shows photo-active responsiveness as well as liquid crystalline property. Hence, they might be utilized in optical device technology as potential optical data storage material and also, utilized for flame retardant applications because of their high thermal stability.

\section{References}

[1] B. Samdahl, Diveratrylidene and divanillydene compounds of cyclohexanone, comprising several new indicators, J. Pharm. Chim. 7 (1928) 162-173.

[2] B. Samdahl, Diveratrylidene and divanillydene compounds of cyclohexanone comprising several new indicators, Chem. Abstr. 22 (1928) 3145.

[3] D.G. Borden, C.C. Unruh, S.H. Merrill, United States Patent 3,453,237, Eastman Kodak Company, Rochester, N.Y., 1969.

[4] D.G. Borden, C.C. Unruh, S.H. Merrill, United States Patent 3,647,444, Eastman Kodak Company, Rochester, N.Y., 1972

[5] Gangadhara, K. Kishore, Novel photocrosslinkable liquid-crystalline polymers: poly[bis(benzylidene)] esters, Macromol. 26 (1993) 2995-3003.

[6] M. Murali, A.B. Samui, Photoactive, liquid-crystalline, hyperbranched benzylidene polyesters: Synthesis and characterization, J. Polym. Sci. Part A: Polym. Chem. 44 (2006) 53-61.

[7] M. Murali, A.B. Samui, Bisbenzylidene cycloalkanone: a versatile molecule as a polymer building block, J. Mater. Chem. 20 (2010) 2714-2737.

[8] V. Srinivasa Rao, A.B. Samui, Structure-property relationship of photoactive liquid crystalline polyethers containing benzylidene moiety, J. Polym. Sci. Part A: Polym. Chem. 47 (2009) 2143-2155.

[9] G. Deepa, R. Balamurugan, P. Kannan, Photoactive liquid crystalline polyesters based on bisbenzylidene and pyridine moieties, J. Mol. Struct. 963 (2010) 219227.

[10] K. Balaji, S.C. Murugavel, Synthesis, spectral, and thermal characterization of photosensitive poly(ether-ester)s containing $\alpha, \beta$-unsaturated ketone moieties in the main chain derived from 2,6-bis[4-(3-hydroxypropyloxy)-3methoxybenzylidene]cyclohexanone, J. Appl. Polym. Sci. 120 (2011) 31413150.

[11] R.W. Lenz, J.I. Jin, Liquid crystal polymers. 3. Thermotropic rigid aromatic copolyesters with bisphenol spacers, Macromol. 14 (1981) 1405-1411. 
[12] R. Cai, E.T. Samulski, Liquid-crystalline aromatic polyesters containing isophthalic acid, Macromol. 27 (1994) 135-140.

[13] S.M. Hong, J. Economy, Effect of annealing on the physical properties of biaxially oriented liquid crystalline copolyester films, Macromol. 28 (1995) 6481-6487.

[14] K. Shigehara, H. Sano, E. Tsuchida, Photogalvanic effects of thin-layer photocells composed of thionin-polymer/Fe (II) systems, Makromol. Chem. 179 (1978) 1531-1539.

[15] A. Reiser, Photoreactive polymers - The science and technology of resist, Wiley, New York, 1989.

[16] S. Tazuke, Developments in polymer photochemistry, Applied Science Publishers, London, 1982.

[17] K. Subramanian, V. Krishnasamy, S. Nanjundan, A.V. Rami Reddy, Photosensitive polymer: synthesis, characterization and properties of a polymer having pendant photocrosslinkable group, Eur. Poly. J. 36 (2000) 2343-2350.

[18] V. Kannappan, P. Sathyamoorthi, D. Roopsingh, Synthesis and characterization of certain thermotropic liquid crystalline random copolyesters containing azobenzene moiety in the main chain, J. Polym. Mater. 19 (2002) 65-74.

[19] K.S. Khairou, M.A. Abdullah, K.I. Aly, N.M. Nahas, A. Al-Bonian, Synthesis and characterization of new poly(ester-amide)s containing diarylidenecyclohexanone in the main chain, Part - II, Arabian J. Chem. 2 (2009) 65-71.

[20] V. Kannapan, D. Reuben Jonathan, A study on the synthesis and bactericidal efficacy of certain poly(ester-amides) containing 2,5bis(benzylidene)cyclopentanone moiety in the main chain, J. Chem. Pharm. Res. 5 (2013) 382-386.

[21] S.M. Aharoni, Hydrogen-bonded highly regular strictly alternating aliphaticaromatic liquid-crystalline poly(ester amides), Macromol. 21 (1988) 19411961.
[22] H. Sang-II, K. Byung-Soo, K. Sun-Woong, S. Hirofusa, S.I. Seung, Cellular interactions and degradation of aliphatic poly(ester amide)s derived from glycine and/or 4-amino butyric acid, Biomater. 24 (2003) 3453-3462.

[23] E. Arumugasamy, Ph.D. Thesis, University of Madras, Chennai, India, 1998

[24] V. Kannapan, E. Arumugasamy, E. Ravichandran, B. Baskar, Synthesis and characterization of certain thermotrophic liquid crystalline random copolyesters, J. Polym. Materials 17 (2000) 4-9.

[25] J. Arul Moli, S. Vasanthi, N. Prakash, D. Roop Singh, Synthesis and characterization of certain photocrosslinkable thermotropic liquid crystalline random copolyesters containing arylidene moiety, High Perform. Polym. 24 (2012) 507-520.

[26] P. Rajakumar, K. Ganesan, S. Jayavelu, K. Murugesan, Synthesis and bactericidal efficacy of novel dendrimers, Syn. Lett. 7 (2005) 1121-1124.

[27] P. Sathish, M. Mayavathi, D. Reuben Jonathan, D. Roopsingh, Synthesis and characterization of certain copolyester PVC-nanoclay composites possessing arylidene-cyclohexanone moiety in the main chain, Int. J. Innov. Res. Sci. Engg. 2 (2014) 1-5.

[28] M. Nagata, M. Nakae, Synthesis, characterization and in vitro degradation of thermotropic polyesters and copolyesters based on terephthalic acid, 3(4hydroxyphenyl)propionic acid and glycols, J. Polym. Sci. Part A: Polym. Chem. 39 (2001) 3043-3051.

[29] T.S. Perundevi, D. Reuben Jonathan, S. Kothai, Synthesis and characterization of certain photocrosslinkable random copolyesters with bischalcone moiety, Int. J. Adv. Res. 3 (2015) 1147-1154.

[30] J. Suresh, S. Karthik, A. Arun, Photocrosslinkable polymer based on 4-(3-(2,4dichlorophenyl)-3-oxoprop-1-enyl) phenylacrylate: synthesis, reactivity ratio, and crosslinking studies, Mater. Sci. Poland 34 (2016) 834-844. 